\title{
ANÁLISE DA COMPETITIVIDADE DOS ESTALEIROS NACIONAIS FABRICANTES DE EMBARCAÇÕES OFFSHORE ATRAVÉS ESTUDO DE CASOS MÚLTIPLOS
}

\section{A COMPETITIVITY ANALYSIS OF BRAZILIAN OFFSHORE SHIPYARDS THROUGH CASE STUDY TECHNIQUE}

\author{
Luiz Carlos Brasil de Brito Mello* E-mail: luiz.brasil@gmail.com \\ Renata Albergaria de Mello Bandeira** E-mail: re.albergaria@gmail.com \\ José Rodrigues de Farias Filho* E-mail: fariasfilho@gmail.com \\ *Universidade Federal Fluminense, Niterói,RJ \\ ${ }^{* *}$ Instituto Militar de Engenharia, Rio de Janeiro, RJ
}

Resumo: $O$ artigo apresenta, através do estudo de casos múltiplos, uma análise comparativa da operação de estaleiros nacionais frente a estaleiros estrangeiros selecionados como benchmarks. A escolha destes benchmarks, assim como a dos estaleiros nacionais, foi feita com o apoio de nove executivos dos três maiores clientes do mercado nacional. $\mathrm{O}$ escopo da pesquisa consiste em identificar quais as práticas gerenciais adotadas pelos estaleiros estrangeiros mais bem sucedidos devem ser implantadas pelos estaleiros domésticos, de modo a torná-los globalmente competitivos. Adotou-se a técnica de análise de conteúdo e, como resultado, analisou-se o desempenho dos estaleiros fabricantes de embarcações offshore em relação às seguintes dimensões: (i) métodos e técnicas de produção; (ii) arranjo físico; (iii) utilização de tecnologia de informação; (iv) gestão estratégica; (v) planejamento e controle da produção; (vi) utilização de ferramentas de apoio ao projeto e (vii) gerenciamento da cadeia de suprimentos. A pesquisa contribui para a prática gerencial ao propor um conjunto das melhores práticas a serem adotadas pelos estaleiros nacionais de modo a melhorar seu desempenho.

Palavras Chave: Estaleiros. Gestão. Benchmark. Estudo de caso.

Abstract: The article aims to compare, through the assessment of multiple cases studies, the domestic shipyards operations against foreign shipyards that were selected as benchmark. The selection of these benchmarks, as well as the national shipyards, was made through interviews with nine executives from the three of largest industry customers in Brazil. The purpose of the research is to identify which management approaches adopted by the most successful foreign yards must be implemented on domestic yards in order to make them internationally competitive. The content analysis technique was adopted in the interview assessment. As a result, the performance of yards manufacturers of oil platforms and support vessels for offshore exploration was measured according to the following dimensions: (i) methods and production techniques; (ii) layout; (iii) use of information technology; (iv) strategic management, (v) planning and production control, (vi) design support tools and (vii) supply chain management. The research contributes to management practice by providing a set of best practices to be adopted in order to domestic shipyards improve their performance.

Keywords: Shipyards. Management. Benchmark. Case study technique. 


\section{INTRODUÇÃO}

A economia brasileira tem passado, nas duas últimas décadas, por transformações, saindo de um longo marasmo com alta inflação e poucos investimentos para um período com estabilidade da inflação e incremento nos investimentos, principalmente após a abertura da economia e o fim da reserva de mercados (BONELLI; PINHEIRO, 2008). Nesta nova economia, marcada por acirrada competitividade, empresas brasileiras buscam ganhos de produtividade por meio de inovações tecnológicas e pela adoção de novos modelos organizacionais, formando um núcleo dinâmico e moderno (GONÇALVES, 2008). A presença de empresas brasileiras no exterior, como a Vale, Petrobrás e AmBev, é prova da capacidade técnica e financeira destes grupos empresariais.

Uma das áreas da economia brasileira que mais evolui nos últimos anos é a indústria do petróleo e gás (ÚLTIMO SEGUNDO, 31/08/09), que passa por processo de diversificação devido ao aquecimento global do setor e a descoberta das reservas do pré-sal. Esta indústria apresenta uma crescente demanda por embarcações de grande porte e velocidade, tal como navios de apoio e plataformas para a exploração das novas reservas petrolíferas brasileiras (DE NIGRI et al., 2009). Contudo, para atender a demanda nacional, é preciso que os estaleiros nacionais apresentem vantagens competitivas em relação a seus concorrentes estrangeiros. Portanto, o governo federal decidiu investir para aumentar a competitividade deste segmento. Empresas nacionais e estrangeiras também têm investido na capacitação do parque industrial, na qualificação de profissionais e aquisição de tecnologias.

Apesar do atual processo de modernização do setor, os estaleiros nacionais ainda precisam atingir um nível de excelência operacional compatível ao observado em países desenvolvidos (DE NIGRI et al., 2009). Neste contexto, esta pesquisa busca responder a seguinte questão: quais práticas gerenciais adotadas por estaleiros estrangeiros bem sucedidos devem ser implantadas pelos estaleiros domésticos, de modo a torná-los globalmente competitivos? São estabelecidas comparações, por meio de estudos de caso múltiplos, entre um grupo de estaleiros nacionais vis a vis à operação de um grupo de estaleiros estrangeiros, considerados organizações de classe mundial. Os estaleiros analisados (brasileiros e benchmarks) 
operam na construção e reparo e embarcações offshore. Procurou-se determinar como os estaleiros nacionais operam e, principalmente, identificar práticas gerenciais que devem ser adotadas para aumentar sua competitividade. Ainda, considerando que o Brasil atravessa uma fase de crescimento, os resultados obtidos também podem servir de ponto de partida para que outros países em desenvolvimento se utilizem de tais técnicas na sua indústria de construção naval.

\section{A INDÚSTRIA DE CONSTRUÇÃO NAVAL BRASILEIRA}

A construção naval tem sido o tema central de inúmeras pesquisas. Craggs et al. (2004) e Collins e Pinto (2006) estudaram aspectos financeiros desta indústria e concluíram que os estaleiros com os melhores resultados financeiros utilizavam as melhores práticas gerenciais. Por outro lado, Gordon (2000), Lahnstein (2004) e Sedler (2005) direcionaram suas pesquisas para aspectos não financeiros, obtendo a conclusão de que os estaleiros japoneses, coreanos e chineses possuem as melhores estratégias de produção, capacidade gerencial e produtividade. Sedler (2005) também afirma que os estaleiros americanos e europeus estão perdendo posições no ranking competitivo.

A indústria da construção naval brasileira tem sido estudada por autores como Correa (1993), Grassi (1995), Takimoto (2005), Pereira e Laurindo (2007) e Moura (2008). Correa (1993) analisou a organização produtiva dos estaleiros nacionais. Grassi (1995) apresenta uma análise histórica da indústria entre 1958 e 1994. Takimoto (2005) estudou a estrutura organizacional de um estaleiro público nacional, apresentando meios para otimizar sua eficiência. Pereira e Laurindo (2007) abordaram o uso e o impacto da Tecnologia da informação (TI) na indústria da construção naval brasileira. Moura (2008) avaliou fatores críticos de sucesso relativos a custo, qualidade e prazos, concluindo que os estaleiros nacionais têm problemas devido à falta de integração, qualificação profissional e produtividade. Assim, o escopo do presente artigo é identificar os fatores determinantes para a diferença de resultados entre os fabricantes offshore nacionais em relação aos estrangeiros.

A indústria da construção naval tem evoluído, desde a década de 70 , da fase do stick building (técnica de construção onde não se utilizam conceitos de préRevista Produção Online. Florianópolis, SC, v.11, n. 2, p. 369-398, abr./jun., 2011 
fabricação ou modularização) para a construção orientada para produtos (CII, 2006). Assim, houve uma redução dos custos de construção, aumento da produtividade e dos lucros. Tais resultados foram alcançados principalmente pela indústria da construção naval asiática, onde países como Coréia do Sul, Japão e China detêm $80 \%$ do mercado global. Neste período, a indústria brasileira de construção naval entrou em grave crise com reduções na força de trabalho e problemas na administração do Fundo de Marinha Mercante (FMM). Logo, o Banco de Desenvolvimento Econômico e Social (BNDES) assumiu, na década de noventa, a administração do FMM com intuito de: revitalizar a cadeia de fornecedores do segmento naval; aumentar a produtividade; incentivar a melhoria do parque fabril; e capacitar a mão de obra (BNDES, 2002). Com a expansão da exploração dos recursos petrolíferos na plataforma continental brasileira, abriu-se uma nova fase para a indústria de construção naval, gerando demanda por plataformas de petróleo e navios de apoio, além da realização de obras de reparo e revitalização de plataformas antigas (FOLHA DE SÃO PAULO, 2008).

A indústria da construção naval é verticalizada, sendo também intensiva em mão de obra, apresentando efeitos a montante da cadeia produtiva - como na geração de empregos e renda - que motivam investimentos no setor (FRAGA, 2002). No entanto, para que tais reflexos positivos aconteçam, é necessária a existência de infra-estrutura adequada e mão de obra qualificada, bem como o atendimento de requisitos fundamentais como prazo, custo e qualidade. Outra característica marcante do setor é a extensa gama de empresas atuantes, com portes variados (BNDES, 2010). Os principais estaleiros nacionais localizam-se no Estado do Rio de Janeiro, destacando-se o Estaleiro Ilha (na capital), o Mauá-Jurong (Niterói), o Promar (Niterói) e o Brasfels (Angra dos Reis). Estes estaleiros caracterizam-se, de forma geral, pela grande capacidade de processamento e qualidade técnica, possibilitando a produção de plataformas de exploração de petróleo, navios-tanque de grande porte e embarcações de apoio marítimo.

Contudo, ainda existem diferenças entre a operação dos estaleiros nacionais e daqueles considerados os melhores do setor. Estas diferenças são os principais motivadores para o gap competitivo existente. Há décadas os estaleiros benchmarks utilizam ferramentas e técnicas como automação, construção orientada ao produto, gestão da cadeia de suprimentos, gestão de custos e utilização integrada de Revista Produção Online. Florianópolis, SC, v.11, n. 2, p. 369-398, abr./jun., 2011 
sistemas de informática. Porém, faz pouco tempo que os estaleiros nacionais começaram a adotar tais técnicas (BNDES, 2010). A indústria doméstica precisa de um processo de modernização de modo a atingir a excelência operacional e níveis de desempenho semelhantes aos observados nos países desenvolvidos. Assim, o objetivo da presente pesquisa consiste em determinar as práticas gerenciais adotada por estaleiros de classe mundial que devem ser implantadas pelos estaleiros domésticos com intuito de torná-los globalmente competitivos. A metodologia de pesquisa aplicada é apresentada a seguir.

\section{METODOLOGIA DE PESQUISA}

A presente pesquisa utilizou procedimentos qualitativos, sendo adotado o estudo de caso como estratégia de investigação. Para Yin (2001), estudos de caso múltiplos são indicados para descrever um fenômeno, construir ou testar uma teoria. Logo, justifica-se a utilização desta estratégia de investigação para esta pesquisa, cujo escopo consiste em determinar como os estaleiros nacionais se posicionam no mercado em comparação a estaleiros internacionais de classe mundial, escolhidos como benchmarks.

Inicialmente, foram realizadas entrevistas com nove executivos que atuam nos três maiores clientes nacionais de embarcações offshore. Estes executivos foram questionados sobre o desempenho operacional de seus fornecedores, sendo solicitados a classificá-los quanto ao seu grau de sucesso em atividades de construção de embarcações offshore. A pesquisa considera a definição de sucesso proposta por Davenport (1998), que relaciona o sucesso aos benefícios potenciais obtidos e aos custos associados para atingi-los. Situações de processos de construção "bem" ou "mal sucedidos" foram selecionadas segundo as recomendações de Pettigrew (1990): (i) o fenômeno deve ser observável; (ii) os casos devem ilustrar exemplos de alto e baixo desempenho; e (iii) os casos devem ser compatíveis ao fenômeno analisado. Tais critérios foram utilizados na seleção das organizações estudadas, procurando equilibrar o número de casos de sucesso e de insucesso. Ao final, a amostra de casos de sucesso foi composta por quatro organizações internacionais de classe mundial, reconhecidas no mercado como benchmarks. O grupo nacional é formado por cinco organizações. As empresas 
analisadas são fabricantes de embarcações offshore, sendo suas características apresentadas na seção 5.1 .

O desenvolvimento da investigação ocorreu entre Janeiro e Dezembro de 2009. Inicialmente, foram identificadas dimensões de análise, por meio de pesquisa bibliográfica, para desenvolvimento do estudo comparativo entre os dois grupos de estaleiros. Em seguida, foram realizadas entrevistas semi-estruturadas, segundo roteiro baseado nas dimensões de análise selecionadas e validado por especialistas. As entrevistas tiveram duração média de uma hora e foram gravadas em áudio. Foram entrevistados 18 executivos que trabalham nos nove fabricantes de embarcações offshore analisados. Também foram realizadas visitas guiadas às instalações das empresas estudadas, que foram registradas em vídeo e fotos.

A técnica de análise de conteúdo foi adotada na avaliação das entrevistas. Os resultados confirmaram nas dimensões de análise (temas) definidas a priori. Logo, a operação dos estaleiros foi analisada a partir das seguintes dimensões: métodos e técnicas de produção; arranjo físico; adoção de TI; gestão estratégica; planejamento e controle da produção; utilização de ferramentas de apoio ao projeto; e gestão da cadeia de suprimentos. Foi desenvolvido um estudo comparativo entre os dois grupos de estaleiros com a finalidade de identificar as dimensões determinantes para o nível de sucesso obtido pelos estaleiros estudados e que corroboram para as diferenças encontradas entre os dois grupos. A seção seguinte apresenta a introdução teórica às dimensões consideradas na pesquisa.

\section{INTRODUÇÃO TEÓRICA AOS ASPECTOS CONSIDERADOS NA PESQUISA}

A seguir apresenta-se a fundamentação teórica das dimensões de análise que guiaram o desenvolvimento desta pesquisa.

\subsection{Métodos e técnicas de produção}

A indústria da construção naval caracteriza-se por executar um processo de manufatura complexo, que se compõe de diversos conjuntos, organizados em diferentes sistemas funcionais. O gerenciamento destes sistemas requer um perfeito controle de identificação das partes e peças, bem como o monitoramento da 
execução de tarefas. Portanto, é necessária a divisão do processo em suas partes componentes para facilitar o controle (STORCH et al., 1995). Tal divisão é conhecida como estrutura de divisão de serviços. A estrutura de divisão de serviços utilizada na construção naval pode ser orientada por sistemas ou por produtos. Atualmente, a construção naval é baseada em blocos cada vez maiores, com vários equipamentos para aumentar a velocidade de construção do navio, de modo que a divisão por produtos tem se mostrado como um importante fator para melhorar a produtividade (TAKIMOTO, 2005).

Também se utiliza, na construção naval, o método de produção conhecido como "projeto voltado para a produção", que consiste na utilização de técnicas de projeto e produção que objetivam reduzir a quantidade de trabalho e atingir as especificações de projeto e qualidade. A utilização deste conceito traz aumentos de produtividade que podem ser alcançados sem maiores investimentos (LAMB, 1986). Os melhores players mundiais da construção naval costumam adotar a Tecnologia de Grupo, que consiste no arranjo lógico e sequenciado de todas as facetas de operação de uma organização para trazer os benefícios da produção em massa, permitindo uma produção de alta variedade (LAMB, 2005).

Atualmente, os estaleiros mais modernos utilizam técnicas de lean manufacturing (do inglês, manufatura enxuta), que se baseiam nos princípios norteadores do Sistema Toyota de Produção. O principal objetivo deste sistema consiste na entrega de um produto de alta qualidade, com baixo custo e no menor tempo, através da eliminação dos desperdícios durante o processo (WOMACK; JONES, 2003). Os princípios de manufatura enxuta não devem ser aplicados somente na produção, mas em toda cadeia produtiva. Ainda, a filosofia de manufatura enxuta não alcançará seu potencial total se não for acompanhada de outros elementos como: Just in time, controles visuais, kaizen, dispositivos à prova de erro, takt time (LIKER, 2005). Storch et al. (1999) afirmam que a manufatura enxuta na construção naval pode ser definida como "um fluxo uniforme e contínuo através da cadeia de valor de cada um dos sistemas cobrindo cada uma das etapas de fabricação do navio". 


\subsection{Arranjo físico}

A indústria da construção naval é muito antiga, de modo que as técnicas construtivas têm evoluído ao longo do tempo devido aos avanços de projeto, materiais, marketing e aspectos construtivos (LAMB, 2005). Até a década de 1970, os fabricantes continuavam a empregar a filosofia de produção em massa ou linha de produção. Porém, a partir daí, uma nova filosofia de construção naval apareceu e demonstrou ser mais efetiva por razões técnicas e econômicas. Esta nova filosofia se baseava na organização celular e pode ser utilizada nas diferentes partes dos produtos offshore (TAKIMOTO, 2005).

Em um amplo estudo desenvolvido pelo Cll (2006), observou-se que a engenharia de processo é uma das ferramentas de trabalho mais utilizadas pelos estaleiros mais produtivos. A engenharia de processo consiste na representação gráfica da sequência de montagem dos componentes do empreendimento. Para cada bloco, é preparada a formalização dos estágios de produção, utilizada para determinar o fluxo ótimo de montagem. Assim, o fluxo pode ser estudado dentro da seguinte orientação: Produto final, montagem dos blocos, submontagens, fabricação das peças (CII, 2006). Após o levantamento desta representação gráfica, é possível, através do estudo do arranjo físico, garantir a proximidade das estações de trabalho, tornando mais eficientes as rotas, criadas pelo sistema de planejamento e controle da manufatura, bem como facilitar a movimentação de pessoas e materiais através do sistema.

Torres (2001) destaca as principais vantagens obtidas pela otimização do arranjo físico: eliminação do estoque central, transferindo a responsabilidade pela estocagem para as células onde são fabricadas, através da criação de estoques entre as células; melhor utilização dos equipamentos, mão-de-obra e serviços, através da redução das distâncias e dos tempos improdutivos; maior qualidade através do melhor posicionamento de equipamentos; agregação dos processos dedicados a família de produtos em torno do processo final, para minimizar a distância de transporte dos componentes dentro da fábrica; economia de espaço, minimizando o nível de material em processo, diminuindo distâncias e dispondo as seções de maneira racional; maximização da área de contato entre os diversos processos de forma que a recepção e expedição de materiais se dêem o mais 
próximo possível de cada setor; facilitar o balanceamento da produção; e organizar o trabalho do operário, em conjunto com a utilização dos princípios de tempos e métodos, de forma a intensificar a utilização da força de trabalho.

\subsection{Tecnologia de informação}

Novos sistemas de informação são constantemente implantados por diferentes organizações com intuito de melhorar a produção, qualidade e eficiência, tanto das áreas administrativas como de manufatura (LAUDON; LAUDON, 2004; LUTCHEN, 2004). Pereira e Laurindo (2007) ressaltam o papel da TI para que as organizações de construção naval desenvolvam seus projetos de forma mais integrada, possibilitando a otimização do gerenciamento de processos e desenvolvimento de produtos. Os autores (2007) destacam o software de gestão integrada (ERP - Enterprise Resource Planning) como um dos principais sistemas de $\mathrm{TI}$ adotados pela indústria de construção naval.

Os sistemas ERP são adquiridos através de pacotes de software comercial para dar suporte às operações de uma organização (CANHETTE et al., 2005). Tais pacotes são divididos em módulos que se comunicam e atualizam uma base de dados central, sendo cada módulo responsável por uma função específica do sistema. As informações alimentadas em um módulo são disponibilizadas instantaneamente para os demais (ALBRECHT et al., 2005), de modo que o ERP permite a integração das diferentes áreas funcionais (JACOBS; WESTON, 2007). O ERP permite a utilização de ferramentas de planejamento que podem analisar o impacto das decisões de produção, suprimentos, finanças ou recursos humanos em todo o ambiente organizacional. Snider et al. (2009) apresentam as vantagens competitivas de um sistema ERP: (i) aumento de valor percebido pelos investidores e pelo mercado; (ii) visibilidade, base única, informação em tempo real; (iii) atendimento a requerimentos globais, regionais e locais em um único sistema (multilíngüe e multimoeda); (iv) suporte a estratégia de e-business; e (v) agilidade para aproveitar oportunidades de negócio.

A implantação de sistemas complexos como o ERP pode trazer, além dos benefícios esperados, desvantagens e problemas para as empresas. Uma das principais dificuldades encontradas é a necessidade de mudanças organizacionais 
profundas nas empresas. Para Poba-Nzaou et al. (2008), o sucesso da implantação do ERP requer um perfeito alinhamento entre os processos existentes na empresa e o software. A implantação de um sistema ERP, que pode levar até três anos, requer uma gestão orientada por processos e o total comprometimento da alta administração (MELLO et al., 2007).

\subsection{Gestão estratégica}

Hayes e Wheelwright (1984), Hill (1985) e Gregory e Platts (1990) consideram que o desenvolvimento de uma estratégia de manufatura deve ter uma abordagem de cima para baixo e sugerem modelos hierárquicos nos quais a estratégia corporativa rege a estratégia de cada unidade de negócio em particular. Porém, Mintzberg (1994) propõe a existência da estratégia emergente, que tem uma abordagem de baixo para cima. A abordagem atual considera que a estratégia de uma empresa é modelada parcialmente pela análise e escolha dos administradores (cima para baixo) e em parte pela necessidade de adaptação e de aprender fazendo (baixo para cima) (THOMPSON et al., 2008). Desta forma, a estratégia de uma empresa é em parte pró-ativa e em parte reativa, sendo necessário estar sempre disposto a complementar ou modificar os elementos pró-ativos da estratégia por meio de reações a acontecimentos não previstos (MINTZBERG et al., 2003).

Atualmente, as mudanças se processam em grande velocidade, de modo que os líderes empresariais precisam traçar cenários e, para cada um destes, elaborar a estratégia mais apropriada (KOTLER; CASLIONE, 2009). As organizações precisam se adaptar aos novos desafios e paradigmas para não perder competitividade. A competitividade está relacionada com "a capacidade de a empresa formular e implementar estratégias concorrenciais, que lhes permitam ampliar ou conservar, de forma duradoura, uma posição sustentável no mercado" (FERRAZ et al., 1997). Para atingir a posição sustentável sugerida por Ferraz et al. (1997), a estratégia operacional deve se basear no desenvolvimento e manutenção de vantagens competitivas, que dependem basicamente de três elementos críticos: capacidades, práticas e recursos (WU et al., 2010).

Um fator de competitividade só é transformado em vantagem competitiva quando consumidores e clientes reconhecem a posição diferenciada da organização

Revista Produção Online. Florianópolis, SC, v.11, n. 2, p. 369-398, abr./jun., 2011 
em relação a tal fator (KOTLER; CASLIONE, 2009). Assim, para que as organizações sejam competitivas, é importante que disponham de vantagens e competências obtidas através do estabelecimento de estratégias. Para Slack et al. (2009), empresas de sucesso são aquelas que adotam estratégias competitivas adequadas ao padrão do seu setor. Segundo Moura (2008), a indústria da construção naval nacional ainda carece de uma visão clara para o estabelecimento de estratégias competitivas adequadas ao fortalecimento do setor. O autor (2008) destaca que a estratégia utilizada pelos estaleiros nacionais para aumentar sua competitividade no mercado internacional ainda consiste no estabelecimento de parcerias com empresas estrangeiras para prestar o serviço e assistência técnica aos produtos domésticos.

\subsection{Planejamento e controle da produção}

Planejar e controlar são atividades essenciais em qualquer setor industrial. Controlar consiste em identificar e quantificar desvios relativos às previsões originais para que sejam adotadas ações preventivas e corretivas com intuito de atingir os resultados desejados (SRIPRASERT et al., 2002). Na execução de empreendimentos da construção naval, assim como nos demais setores, é necessária a otimização de recursos (materiais, mão-de-obra, equipamentos e capital) finitos, que estão sujeitos a limites e restrições. O fornecimento de dados para o controle e alocação destes recursos no devido tempo requer um sistema de planejamento e programação eficaz (KRAJEWSKI et al., 2009).

Para Groover (2000), um sistema de controle típico de ordens de produção consiste de três fases: (1) liberação; (2) programação; e (3) acompanhamento de ordens. Swink et al. (2011) consideram que o objetivo do controle da produção consiste no acompanhamento da produção realizada para comparar o real ao planejado, sendo suas principais atividades: a) Controlar equipamento, b) Controlar produção de itens, c) Controlar produção de ordens e lotes, e d) Registrar informações de controle. Vollmann et al. (1993) reforçam a importância de gerenciar cada componente, submontagem e produto em todas as etapas da produção, apesar do grande volume de informações requeridas. Wall (1995) também destaca que o controle da produção permite uma visão da situação da produção da empresa.

Revista Produção Online. Florianópolis, SC, v.11, n. 2, p. 369-398, abr./jun., 2011 
Para Slack et al (2009), uma empresa pode obter competitividade a partir da manufatura com base cinco vantagens específicas: Qualidade, Velocidade Confiabilidade, Flexibilidade e Custo. Para uma efetiva introdução de um sistema de manufatura, é indispensável a adoção de um sistema de planejamento e controle do chão de fábrica. Para Junqueira (2003), o sucesso de um sistema de manufatura depende de seis dimensões de informação: mercado, produto, tecnologia de processo, sistema cultural, desempenho da manufatura e requisitos empresariais.

\subsection{Utilização de ferramentas computacionais de apoio ao projeto}

Atualmente, observa-se cada vez mais a utilização de computadores nas diversas atividades da engenharia, pois a melhoria contínua dos equipamentos (hardware) resultou na maior disponibilidade de sistemas computadorizados para manufatura. Os impactos positivos de ferramentas computacionais de apoio ao projeto podem ser alcançados por meio da implementação de banco de dados, de softwares com plataforma CAD/CAE/CAM e de controle de projetos (por exemplo, o Microsoft Project), interconectando e acompanhando os processos de desenvolvimento (LAURINDO; CARVALHO, 2005).

Omer et al. (2006) destacam as ferramentas computacionais mais utilizadas na indústria da construção naval: (i) o Computer-Aided Design - CAD (do inglês, Projeto Auxiliado por Computador): software baseado em interface gráfica orientada ao usuário utilizado para a criação, modificação, análise ou aprimoramento de projetos; (ii) Computer-Aided Engineering - CAE (do inglês, Engenharia Auxiliada por Computador): recursos informatizados para a análise das atividades de engenharia, tais como cálculos de esforços, cálculos de transferência de calor, dentre outros; (iii) Computer-Aided Manufacturing- CAM (do inglês, Fabricação Auxiliada por Computador): sistemas computacionais para planejar, gerenciar e controlar as operações de fabricação através de uma interface com os recursos produtivos; (iv) Computer-Integrated Manufacturing- CIM (do inglês, Fabricação Integrada por Computador): voltado para a disponibilização da informação necessária em toda a empresa. Os autores (2006) reforçam que, atualmente, a indústria da construção naval mundial tem como meta a utilização destas tecnologias, integrando-as no processo produtivo de maneira a atender a tripla restrição dos empreendimentos 
(custo, escopo, prazo) e às necessidades do cliente. Para Pereira e Laurindo (2007), ferramentas como o CAD, CAE e CAM são inovações tecnológicas adotadas pela indústria da construção naval com intuito de melhorar eficiência do desenvolvimento de novos produtos e o processo de fabricação.

O CAD 3D foi, inicialmente, adotado em projetos de engenharia para facilitar a visualização do processo construtivo da embarcação (INTERGRAPH, 2004). Ultimamente, recursos como BIM (Building Information Models) e 4D são utilizados para integrar as práticas de arquitetura, engenharia e construção (WILHELM, 2007). Modelos 4D são CAD 3D com a adição do fator tempo, possibilitando o acompanhamento da evolução do empreendimento ao longo do tempo. BIMs são softwares CAD 3D com databases adicionadas com informações como: propriedades e quantidades dos materiais e dados sobre fabricantes. Assim, além da visualização do empreendimento, a utilização de modelagem BIM e 4D permite a avaliação de ambigüidades e conflitos de projeto em sua fase inicial, possibilitando correções durante o projeto que resultam em economias (INTERGRAPH, 2004). Para Khanzode et al (2006), a utilização das ferramentas de visualização (BIM, 3D, 4D) possibilitam um entendimento comum, entre os participantes do empreendimento, sobre qual será sua configuração final. Wilhelm (2007) reforçam que a utilização de modelagem 3D e 4D em produtos e processos facilita a colaboração entre os participantes da cadeia de valor.

\subsection{Gerenciamento da cadeia de suprimentos}

Recentemente, autores têm atribuído ao gerenciamento da cadeia de suprimentos (Supply Chain Management - SCM) o status de filosofia de negócios, e diversas definições e visões têm sido encontradas na literatura (SVENSSON, 2002). A SCM pode ser considerada uma visão expandida, atualizada e, sobretudo, holística da administração tradicional de materiais, abrangendo a gestão de toda a cadeia produtiva de forma estratégica e integrada (CHOPRA; MEINDEL, 2006; BOWERSOX et al., 2007; SWINK et al., 2011).

A competição não se restringe mais ao nível organizacional, ocorrendo cada vez mais no nível das cadeias produtivas (REUER; ARIÑO, 2007). Logo, torna-se necessário o estreitamento dos relacionamentos entre os participantes da cadeia

Revista Produção Online. Florianópolis, SC, v.11, n. 2, p. 369-398, abr./jun., 2011 
(SCHOENHERR, 2010). Cooper e Gardner (1993) e Bowersox et al. (2007) indicam pontos importantes para o estabelecimento e consolidação dos relacionamentos na cadeia: a) Assimetria, demonstrando a habilidade de uma organização em exercer poder, influenciar ou controlar outra organização, b) Reciprocidade, baseada nos benefícios mútuos para atingir objetivos comuns, c) Eficiência, refletindo a necessidade interna da organização em melhorar a relação custo/benefício de um processo, d) Estabilidade, refletindo a tentativa de adaptar e/ou reduzir as incertezas de algum negócio, e) Legitimidade, justificando as atividades e resultados de uma organização.

O gerenciamento eficaz da cadeia de suprimentos requer constante aperfeiçoamento, visando resultados positivos não somente no âmbito da empresa produtora, mas também para seus fornecedores e para a cadeia em geral. Para Wu et al (2006), a adoção de recursos de TI é um fator importante para o desenvolvimento das capacidades dos participantes da cadeia de suprimentos, aumentando assim a competitividade e eficácia geral da cadeia, já que permite uma melhor coordenação e troca de informações. Contudo, uma perfeita gestão da cadeia de suprimentos também deve considerar fatores como: a) integração de processos de negócio, b) identificação dos membros chave da cadeia, c) compartilhamento de informações e d) adoção de medidas de desempenho (ARAGÃO et al., 2004).

Coutinho et al. (2006) destacam que a cadeia de suprimentos da construção naval passa por transformações consideráveis, como: redução do número de fornecedores diretos e abastecimento na cadeia via sistema just in time. A gestão da cadeia de suprimentos da indústria da construção naval requer a integração entre os elos existentes, de modo a permitir a vantagem competitiva ao setor. Para Moura (2008), a gestão da cadeia de suprimentos naval deve ser capaz de aumentar a competitivade dos componentes da cadeia por meio do compartilhamento de conhecimentos, da intensificação de ferramentas computacionais, do desenvolvimento de novos fornecedores e formação de parcerias.

Nesta seção, foram apresentadas as dimensões analisadas na pesquisa. $O$ Quadro 1 sintetiza as dimensões consideradas e a bibliografia de apoio. 
Quadro 1 - Dimensões analisadas em relação à operação dos estaleiros e bibliografia de apoio

\begin{tabular}{|c|l|}
\hline Dimensão analisada & \multicolumn{1}{|c|}{ Autores } \\
\hline $\begin{array}{c}\text { Métodos e técnicas de } \\
\text { produção }\end{array}$ & $\begin{array}{l}\text { Ramson (1972), Lamb (1986), Womack et al, 1986, Storch et al (1995), } \\
\text { Storch et al (1999), Wolmack e Jones (2003), Liker (2005), Takimoto } \\
\text { (2005). }\end{array}$ \\
\hline Arranjo físico & Torres (2001), Lamb (2005), Takimoto (2005), Cll (2006). \\
\hline $\begin{array}{c}\text { Utilização de } \\
\text { tecnologia de } \\
\text { informação }\end{array}$ & $\begin{array}{l}\text { Lutchen (2004), Laudon e Laudon (2005), Albrecht et al (2005), Canhete } \\
\text { et al (2005), Jacobs e Weston (2007), Mello et al (2007), Pereira e } \\
\text { Laurindo (2007), Poba-Nzaou et al. (2008), Snider et al. (2009). }\end{array}$ \\
\hline $\begin{array}{c}\text { Gestão estratégica } \\
\text { Hayes e Wheelwright (1984), Hill (1985), Gregory e Platts (1990), } \\
\text { Mintzberg (1994), Ferraz et al (1997), Mintzberg et al. (2003), Thompson et } \\
\text { al. (2008), Moura (2008), Kotler e Calione (2009), Slack et al. (2009), Wu } \\
\text { et al. (2010). }\end{array}$ \\
\hline $\begin{array}{c}\text { Planejamento e } \\
\text { contronann et al. (1993), Wall (1995), Groover (2000), Slack et al. (2009), } \\
\text { Sriprasert et al (2002), Junqueira (2003), Krajewski et al. (2009), Swink et } \\
\text { al. (2011). }\end{array}$ \\
\hline $\begin{array}{c}\text { Ferramentas } \\
\text { computacionais de } \\
\text { apoio ao projeto }\end{array}$ & $\begin{array}{l}\text { Oaurindo e Carvalho (2005), Intergraph (2004), Khanzode et al (2006), } \\
\text { Omet al (2006), Pereira e Laurindo (2007), Wilhelm (2007) }\end{array}$ \\
\hline $\begin{array}{c}\text { Gerenciamento da } \\
\text { cadeia de suprimentos }\end{array}$ & $\begin{array}{l}\text { Cooper e Gardner (1993), Svensson (2002), Aragão et al (2004), Wu et al } \\
(2006), \text { Chopra e Meindel (2006), Coutinho et al. (2006) Bowersox et al. } \\
(2007), \text { Reuer e Ariño (2007), Moura (2008), Schoenherr (2010), Swink et } \\
\text { al. (2011). }\end{array}$ \\
\hline
\end{tabular}

Fonte: Elaborado pelos autores

\section{ESTUDO DE CASO}

Nesta seção, é feita uma contextualização das empresas estudadas e, em seguida, é apresentada a análise dos resultados dos casos estudados.

\subsection{Unidades de análise}

As empresas analisadas nos estudos de caso múltiplos solicitaram para não serem identificadas e, por questões de sigilo, dados econômicos e financeiros não são divulgados neste artigo. Foram analisados nove fabricantes de embarcações offshore, sendo cinco localizados no Brasil e quatro no exterior. Os estaleiros brasileiros situam-se no Estado do Rio de Janeiro e Pernambuco, enquanto que os estrangeiros situam-se na Malásia, Coréia do Sul, Noruega e Cingapura. Estes estaleiros, brasileiros e estrangeiros, são organizações de grande porte que fabricam embarcações offshore.

Conforme apresentado na seção 3 , os fabricantes internacionais de embarcações offshore são considerados benchmark, sendo reconhecidos como 
organizações de nível mundial, bem sucedidas e competitivas. O grupo de benchmarks é composto por quatro estaleiros: (i) o estaleiro norueguês tem amplo conhecimento do projeto, gerenciamento, construção, comissionamento e operação de plataformas, navios de apoio e equipamentos para a indústria de petróleo e gás; (ii) o estaleiro malaio é pioneiro no país e tem como áreas de atuação Engenharia e Construção, Reparos Navais e Conversões Marítimas. Possui uma força de trabalho permanente de 1500 funcionários, que é suportada por empresas terceirizadas; (iii) o estaleiro coreano é especializado na construção de plataformas flutuantes e fixas, além de navios de apoio para a indústria petrolífera, sendo reconhecido mundialmente por suas excelentes práticas para o projeto, gerenciamento e construção; e (iv) o estaleiro situado em Cingapura pertence a um dos maiores grupos atuantes no segmento offshore de construção naval, atuando no projeto, construção e reparos de navios e plataformas de petróleo.

Devido à longa crise da indústria brasileira de construção naval, não foi realizado o investimento necessário para o desenvolvimento do setor. Porém, atualmente, a demanda do setor cresce devido à descoberta das reservas pré-sal e a decisões governamentais de estímulo à indústria da construção naval. Contudo, é importante destacar que os fabricantes nacionais, de maneira geral, ainda atuam como linhas de montagem, pois a maioria das partes e componentes continua sendo produzida no exterior.

Dos cinco estaleiros brasileiros analisados, quatro são fluminenses. Três destas empresas fluminenses têm capacidade de processamento e qualidade técnica de produção, possibilitando a construção de plataformas de exploração de petróleo (fixas e flutuantes) e navios-tanque de grande porte. O quarto estaleiro fluminense estudado especializou-se na fabricação de navios de apoio para serviços offshore, também possuindo qualidade técnica de produção. $O$ estaleiro pernambucano tem grande capacidade de processamento, atuando no projeto, gerenciamento, construção e reparos de plataformas e de navios de apoio para a indústria offshore. Possui equipamentos e instalações similares aos melhores da construção naval mundial, tendo contrato de assistência técnica com construtor naval de classe mundial.

Os estaleiros analisados durante o desenvolvimento dos estudos de caso, seja do cluster doméstico ou benchmark, têm capacidade de construir plataformas Revista Produção Online. Florianópolis, SC, v.11, n. 2, p. 369-398, abr./jun., 2011 
de exploração de petróleo (fixas e flutuantes), navios-tanque de grande porte e embarcações de apoio marítimo. Contudo, observaram-se oportunidades de melhoria na operação dos fabricantes domésticos, que podem contribuir para tornálos mais competitivos. A soma da produção dos estaleiros do grupo nacional, em conjunto, equivale a apenas $20 \%$ das unidades manufaturadas pelo estaleiro coreano. As principais diferenças relativas às práticas gerenciais adotadas pelos dois grupos de fabricantes de embarcações offshore são apresentadas a seguir.

\subsection{Análise dos resultados do estudo de caso}

Os resultados da pesquisa são apresentados em duas categorias: (i) dimensões não diferenciais, ou seja, não apontadas como causa da diferença de desempenho entre os dois grupos; e (ii) dimensões que emergiram como diferenciais entre o grupo de estaleiros brasileiros e o de benchmarks. Estas são as práticas gerenciais que devem ser adotadas pelos estaleiros nacionais como tentativa de incrementar seu desempenho e competitividade. O Quadro 2 apresenta o panorama das dimensões analisadas nos estaleiros.

Quadro 2 - Panorama das dimensões analisadas nos estaleiros.

\begin{tabular}{|c|c|c|c|c|c|c|c|c|}
\hline Estaleiro & Localização & \begin{tabular}{|c|} 
Métodos e \\
Técnicas de \\
Produção \\
\end{tabular} & Arranjo Físico & Adoção de TI & $\begin{array}{c}\text { Gestão } \\
\text { estratégica }\end{array}$ & \begin{tabular}{|c|} 
Planejamento e \\
controle da \\
produção \\
\end{tabular} & $\begin{array}{c}\text { Adoção de } \\
\text { ferramentas }\end{array}$ & SCM \\
\hline A & RJ, Brasil & Sim & Não & $\begin{array}{c}\text { Sim, devendo ser } \\
\text { intensificada }\end{array}$ & Sim & Sim & $\begin{array}{c}\text { Sim, devendo ser } \\
\text { intensificada }\end{array}$ & Não \\
\hline B & RJ, Brasil & Sim & Sim & Sim & Sim & Sim & $\begin{array}{l}\text { Sim, devendo ser } \\
\text { intensificada }\end{array}$ & Não \\
\hline $\mathrm{C}$ & RJ, Brasil & Sim & Sim & Sim & Sim & Sim & $\begin{array}{l}\text { Sim, devendo ser } \\
\text { intensificada }\end{array}$ & Não \\
\hline $\mathrm{D}$ & RJ, Brasil & Sim & $\begin{array}{l}\text { Sim, devendo ser } \\
\text { intensificado }\end{array}$ & Sim & Sim & Sim & $\begin{array}{l}\text { Sim, devendo ser } \\
\text { intensificada }\end{array}$ & Sim \\
\hline E & PE, Brasil & Sim & Sim & Sim & Sim & Sim & n.a. & ND \\
\hline $\mathrm{F}$ & Nãoruega & Sim & Sim & Sim & Sim & Sim & Sim & Sim \\
\hline G & Malásia & Sim & Sim & Sim & Sim & ND & ND & ND \\
\hline $\mathrm{H}$ & Coreia do Sul & Sim & Sim & Sim & Sim & Sim & Sim & Sim \\
\hline $\mathrm{I}$ & Cingapura & Sim & Sim & Sim & Sim & Sim & Sim & Sim \\
\hline
\end{tabular}

Fonte: Elaborado pelos autores 


\subsubsection{Dimensões não diferenciais}

Nesta seção, são analisadas as dimensões que, segundo os resultados da pesquisa, não contribuem para explicar a variância de sucesso entre os dois grupos de estaleiros, uma vez que não foram observadas diferenças de adoção quanto a estas dimensões pelos fabricantes de embarcações offshore nacionais e os benchmarks.

O planejamento e controle são atividades essenciais em qualquer ramo de atividade industrial, inclusive no segmento da construção naval. Conforme observado no Quadro 2, os estaleiros nacionais, assim como os benchmarks, se dedicam a este aspecto, adotando sistemas de medição de desempenho e recursos de tecnologia de informação para o planejamento e controle da produção. Logo, este não pode ser considerado um fator diferencial para explicar a variação do desempenho obtido entre os grupos de estaleiros nacionais e benchmarks. $O$ desempenho dos processos de produção é medido por indicadores que são periodicamente revisados e atualizados, de modo a refletir as melhorias atingidas. A tecnologia da informação é adotada como instrumento de planejamento e controle, facilitando a integração entre as diversas áreas das empresas, conforme sugere Snider et al. (2009) ao salientar a importância da TI para a melhoria de processos de negócios, tais como produção, compras ou distribuição.

Uma característica comum aos estaleiros nacionais é a preocupação com a integração das equipes de planejamento e controle às demais equipes da planta. $A$ equipe de planejamento e controle é responsável pela obtenção e análise de todas as informações relativas ao projeto, atendendo assim ao estabelecido por Vollmann et al. (1993) e Swink et al. (2011), que consideram que o controle das atividades de produção está relacionado aos mais detalhados aspectos da manufatura. Uma prática positiva, verificada nos estaleiros brasileiros, refere-se ao gerenciamento e programação, por parte das contratantes, das atividades desenvolvidas por empresas terceirizadas. Assim, evitam-se interferências com outras atividades, além de permitir a atualização constante das atividades do cronograma.

Com relação à adoção de tecnologia de informação, observou-se que todos os estaleiros pesquisados utilizam a TI para obter as vantagens citadas por Laudon e Laudon (2004), Lutchen (2004) e Snider et al. (2009), como: aumento de valor 
percebido pelos investidores e pelo mercado, visibilidade, base única de informação em tempo real e agilidade. O alto grau de utilização da TI, tanto pela força de trabalho quanto para o controle das operações, é possível pela existência de um sistema informatizado totalmente integrado nas unidades analisadas. As organizações pesquisadas possuem sistemas de planejamento, controle da produção e de materiais com disponibilidade de informações online em tempo real, permitindo o compartilhamento de informações por todos os setores, como preconizado por Jacobs e Weston (2007). Todas as organizações pesquisadas utilizam sistemas ERP, tal como destacado por Pereira e Laurindo (2007). Porém, ao contrário do sugerido por Canhete et al. (2005), tais sistemas são customizados, ou seja, desenvolvidos internamente. Apenas dois estaleiros (um nacional e outro benchmark) utilizam sistemas comerciais. A decisão de desenvolver os sistemas internamente é estimulada por peculiaridades intrínsecas à indústria da construção naval. A utilização de ferramentas de planejamento permite a análise do impacto das decisões de produção, suprimentos, finanças ou recursos humanos em todo ambiente organizacional. Porém, a implantação de um sistema ERP, que pode levar até três anos, requer uma gestão orientada por processos e apoio da alta administração (MELLO et al., 2007, POBA-NZAOU et al., 2008). Enfim, ambos os grupos seguem as orientações de Pereira e Laurindo (2007) que ressaltam o papel da TI na integração de projetos das organizações da construção naval.

É importante destacar que, embora tais dimensões - adoção da $\mathrm{Tl}$ e planejamento e controle da produção - não sejam consideradas determinantes na diferenciação do grau de competitividade obtido entre os grupos de estaleiros, isto não significa que elas não sejam importantes para o sucesso organizacional. Para a indústria da construção naval, tai dimensões já não são mais consideradas recursos estratégicos, sendo recursos básicos que trazem igualdade competitiva (FORTE et al., 2006).

\subsubsection{Dimensões diferenciais}

Esta seção apresenta as dimensões que os resultados da pesquisa indicaram como fatores determinantes para discriminar as práticas de sucesso adotadas pelos 
nove fabricantes de embarcações offshore divididos em dois grupos - nacionais e os benchmarks.

Verifica-se, a partir do Quadro 2, que os estaleiros nacionais pesquisados, assim como os benchmarks, consideram a dimensão métodos e técnicas de produção como uma ferramenta de gestão importante para a obtenção de ganhos de produtividade (LIKER, 2005). Ambos os grupos adotam técnicas recomendadas por Lamb (1986) e Storch et al (1999), como work breakdown structure (estrutura analítica de projetos) e projeto voltado para a produção. Tais técnicas não geram vantagem competitiva, uma vez que são amplamente conhecidas e adotada por todos, mas agregam valor ao trabalho por permitir maior controle das atividades desempenhadas. No entanto, também são observadas diferenças entre os métodos e técnicas adotados pelos diferentes grupos. Os estaleiros benchmarks são usuários de técnicas de lean manufacturing, porém, apenas um dos estaleiros nacionais utiliza, de forma parcial, esta técnica. Este estaleiro possui um projeto para implantála em toda sua cadeira produtiva, mas os demais estaleiros nacionais não utilizam técnicas de produção enxuta e nem têm planos de adotá-las. Técnicas de produção enxuta já são de amplo conhecimento na indústria nacional em segmentos como a automobilística e o de autopeças, de modo que os estaleiros brasileiros estão defasados em relação a este aspecto. A adoção de práticas de produção enxuta, como Just-in-Time, Kaizen (do inglês, melhoria contínua), Poka-Yoke e Zero-defeito, pela indústria da construção naval pode contribuir para a redução das sete perdas fundamentais e para o aumento da produtividade (SPEAR; BOWEN, 1999; SPEAR, 2004).

Os fabricantes nacionais de embarcações offshore também estão atrasados em relação ao grau de automatização da produção, que é muito avançado nos estaleiros internacionais com utilização intensiva em ambientes confinados de robôs e de gabaritos para facilitar o trabalho. Os estaleiros nacionais devem adotar a automatização de maneira a melhorar as condições de trabalho e segurança na indústria (LIKER, 2005). Assim, a intensificação do grau de automatização na construção naval gerará um aumento da produtividade e qualidade com menor desgaste físico dos trabalhadores.

Todos os estaleiros benchmark consideram o arranjo físico e a localização em seu processo de planejamento, seguindo os preceitos de autores como Torres 
(2001), Takimoto (2005), Lamb (2005) e Cll (2006). Porém, apenas um estaleiro nacional segue tais recomendações. A adoção de tais conceitos pelos estaleiros benchmarks garante a proximidade das estações de trabalho, tornando mais eficientes as rotas criadas pelo sistema de planejamento e controle da manufatura. Logo, o planejamento do arranjo físico e localização das plantas é uma prática gerencial que, ao ser implantado pelos estaleiros nacionais, possibilitará a redução das perdas por movimentação e transporte, bem como o aumento da produtividade.

Todas as organizações pesquisadas - nacionais e benchmarks - utilizam a gestão estratégica em busca de competitividade e de vantagens e competências. Contudo, a cultura dos estaleiros nacionais considera que o acerto de sua gestão estratégica é comprovado pelas certificações obtidas, como ISO 9001 e ISO 14001. Porém, tais certificações são representativas de modalidades de gestão, sejam elas de produção, operação, projetos, recursos humanos ou financeiras, não substituindo efetivamente a estratégia. Segundo Mintzberg (2003) e Thompson et al (2008), empresas de sucesso adotam estratégias competitivas adequadas ao padrão do seu setor. Ainda, tais estratégias só são efetivas quando consumidores e clientes reconhecem a posição diferenciada da organização em relação a seus concorrentes (KOTLER; CASLIONE, 2009). Isto ocorre no grupo de estaleiros benchmarks, uma vez que seus clientes os apontaram como organizações que possuem altos padrões de desempenho e qualidade. No entanto, os fabricantes nacionais ainda carecem de uma visão clara sobre o estabelecimento de estratégias competitivas adequadas ao fortalecimento do setor, conforme destacado por Moura (2008).

Quanto à dimensão utilização de ferramentas computacionais, observouse que os estaleiros benchmark estão mais bem posicionados em relação aos nacionais, pois utilizam com competência recursos como CAD, CAE, CAM e CIM. Os estaleiros nacionais precisam aprofundar a utilização destas ferramentas, cuja adoção tem impactos positivos no desenvolvimento de novos produtos e na gestão de projetos (TAKIMOTO, 2005). Foi constado que os estaleiros nacionais utilizam o CAD 3D em projetos de engenharia para facilitar a visualização, mas não utilizam recursos computacionais de apoio ao projeto como o BIM e 4D na integração das práticas de arquitetura, engenharia e construção. A indústria da construção naval brasileira deve adotar a modelagem 3D e 4D em produtos e processos para facilitar a colaboração entre os participantes da cadeia de valor (WILHELM, 2007). Os 
estaleiros nacionais precisam entender que a indústria global da construção naval integra a utilização destas tecnologias computacionais ao processo produtivo para atender a restrições de custo, escopo, prazo e às necessidades dos clientes (OMER et al., 2006).

O resultado da pesquisa indicou que apenas um dos estaleiros nacionais apresenta um bom gerenciamento da cadeia de suprimentos, com práticas que se equiparam às utilizadas pelas organizações benchmarks. No entanto, o planejamento e controle dos demais estaleiros domésticos não integram a cadeia de fornecimento na extensão necessária, de modo que não permitem uma antecipação em tempo real de ações corretivas nos principais itens de fornecimento. Os administradores deveriam considerar as recomendações de Schoenherr (2010) e Swink et al. (2011) que afirmam que o gerenciamento da cadeia deve abranger a gestão de toda a cadeia produtiva de forma estratégica e integrada.

Os estaleiros benchmarks se dedicam ao aperfeiçoamento da gestão da cadeira de suprimentos, confirmando a importância dada a este conceito por diversos autores - Svensson (2002), Aragão et al. (2004), Bowersox et al. (2007), Moura (2008), Schoenherr (2010). Nestas organizações, a gestão de estoques é computadorizada, sendo disponíveis informações on line sobre o status dos equipamentos e materiais, bem como o acompanhamento de seu ciclo de vida. Ainda, tais estaleiros integram seus sistemas de informação aos de seus principais fornecedores, o que não ocorre nos fabricantes nacionais. A integração entre os elos da indústria nacional de construção naval permitiria um estreitamento dos relacionamentos entre os participantes da cadeia (SCHOENHERR, 2010). Portanto, os estaleiros nacionais devem envidar esforços para o compartilhamento de conhecimentos, a intensificação da utilização da TI no gerenciamento da cadeia e o desenvolvimento de novos fornecedores, com intuito de incrementar a competitividade da cadeia de suprimentos naval.

Ainda, um planejamento detalhado das atividades de engenharia é fundamental para possibilitar um melhor gerenciamento da cadeia suprimentos., uma vez que o SCM é impactado na medida em que os materiais e equipamentos necessários não são definidos de forma plena, no momento devido. Essa prática impossibilita que sejam efetuadas aquisições eficazes, especificações de compras mais precisas, uma seleção adequada de fornecedores, gerando incremento de 
carga gerencial, retrabalho das equipes e potencial prejuízo financeiro. Assim, os estaleiros nacionais precisam seguir o exemplo dos benchmarks, que consideram a integração do planejamento e controle organizacional ao da cadeia como uma importante fonte de competitividade, que contribui para desempenho operacional, redução de prazos, integração de processos e eliminação de gargalos.

\section{CONSIDERAÇÕES FINAIS}

A indústria da construção naval brasileira ressurgiu, após um período de declínio, devido às necessidades da indústria de petróleo. Em um mundo globalizado, as empresas do segmento precisam estar bem preparadas, aumentando sua competitividade para enfrentar a concorrência externa. Portanto, através de estudos de casos múltiplos, analisou-se o desempenho operacional e as práticas gerenciais de cinco estaleiros nacionais em relação a um grupo de quatro estaleiros estrangeiros, reconhecidos como organizações de classe mundial e considerados benchmarks. As empresas analisadas possuem experiência na fabricação de plataformas e embarcações de apoio offshore.

A metodologia de estudos de casos múltiplos permitiu a identificação de fatores determinantes para melhorar a operação dos estaleiros nacionais, aumentando seu desempenho e competitividade. A pesquisa buscou responder a seguinte questão: quais práticas gerenciais adotadas por estaleiros estrangeiros bem sucedidos devem ser implantadas pelos estaleiros domésticos, de modo a torná-los competitivos internacionalmente? Portanto, foram identificadas as diferenças existentes entre a operação dos estaleiros nacionais e daqueles considerados benchmarks com relação a sete dimensões definidas a priori: (i) métodos e técnicas de produção; (ii) arranjo físico; (iii) adoção de TI; (iv) gestão estratégica; (v) planejamento e controle da produção; (vi) utilização de ferramentas computacionais de apoio ao projeto; e (vii) gestão da cadeia de suprimentos. A análise efetuada permitiu que fosse obtida uma visão bastante abrangente da atual situação dos fabricantes de embarcações offshore nacionais em relação estes aspectos, identificando oportunidades de melhoria. Para os autores, as maiores falhas são encontradas em relação às seguintes dimensões: 
a) Métodos e técnicas de produção: em relação a esta dimensão, os benchmarks apresentam desempenho superior aos nacionais, que devem avaliar as vantagens da adoção dos princípios da manufatura enxuta, já aplicados com vantagens em outros ramos industriais;

b) Arranjo físico: esta dimensão ainda é incipiente no grupo de estaleiros nacionais. Esta é uma oportunidade de melhoria para a indústria nacional de construção naval, pois estudos sobre o arranjo físico possibilitam o planejamento da disposição dos centros de trabalho de modo a suavizar e facilitar a movimentação de pessoas e materiais, permitindo aumento de produtividade a partir da redução de perdas por movimentação e transporte;

c) Utilização de ferramentas computacionais de apoio ao projeto: os estaleiros benchmark estão mais bem posicionados em relação a esta dimensão, enquanto os estaleiros nacionais precisam aprofundar a utilização de tais ferramentas. A aplicação de tais ferramentas facilita o desenvolvimento de novos produtos e o gerenciamento de projetos, além de permitir maior integração de informações e atividades, aumentando assim a capacidade dos estaleiros domésticos em melhorar sua competitividade;

d) Gerenciamento da cadeia de suprimentos: esta é a dimensão de pior desempenho dos estaleiros nacionais em relação ao grupo de benchmarks. É preciso que os estaleiros domésticos priorizem a integração dos elos de sua cadeia de suprimentos, planejando a integração de seus sistemas de informação aos de seus principais fornecedores, o compartilhamento de conhecimentos e o desenvolvimento conjunto de novos produtos e de melhorias no processo, visando o aumentar a competitividade em toda a cadeia de suprimentos da construção naval.

Enfim, esta pesquisa apresenta o estágio atual dos fabricantes de embarcações offshore brasileiros em comparação aos internacionais de classe mundial. A pesquisa também tem como contribuição a identificação de um conjunto de dimensões determinantes para o sucesso obtido pelos fabricantes internacionais de embarcação offshore no processo de construção de plataformas de petróleo e 
navios de apoio. A implantação destas práticas gerenciais, por parte dos fabricantes nacionais, contribuirá para aumentar seu desempenho operacional e, conseqüentemente, os níveis de competitividade e de sucesso organizacional. Contudo, é importante ressaltar que, neste estudo, não foram considerados pontos importantes para o aprimoramento da indústria da construção naval brasileira como: carga tributária aplicada, capacitação da mão de obra atuante no setor, aprimoramento dos fornecedores da construção naval dentre outros que merecem ser objeto de trabalhos futuros.

\section{REFERÊNCIAS}

ALBRECHT, G. \& PEDRON, C. Sistema ERP: um estudo de caso sobre cutover. Curitiba: Anais do CATI, 2005.

ARAGÃO, A.; SACAVARDA, L.: HAMACHER, S. Modelo de análise de cadeia de suprimentos: fundamentos e aplicação às cadeias de cilindros de GNV, Gestão e Produção, v.11, n.3, p.299-311, set./dez. 2004.

BNDES. Revista do BNDES. Rio De Janeiro, v. 9, n. 18, p. 121-148, dez. 2002.

\section{Desenvolvimento da cadeia produtiva de petróleo e gás e}

investimentos em E\&P, 2010. Disponível em: < www.bndes.gov.br > . Acesso em: 7 fev. 2010.

BONELLI, R.; PINHEIRO, A.C. Abertura e crescimento econômico no Brasil. In: BARROS, O. ; GIAMBIAGI, F. Brasil globalizado. Rio de Janeiro: Campus, 2008.

BOWERSOX, D.; CLOSS, D. Logística empresarial, o processo de integração da cadeia de suprimento. São Paulo: Atlas, 2007.

CANHETTE, C.; MORAES, R.; GONÇALVES, R. Característica do ciclo de vida de sistemas ERP. Anais do CATI, 2005.

CHOPRA, S.; MEINDL, P. Gerenciamento da cadeia da suprimentos: estratégia, planejamento e operação. São Paulo: Prentice Hall, 2006.

CONSTRUCTION INDUSTRY INSTITUTE, Examination of the shipbuilding industry, Research Report, Austin: University of Texas at Austin, 2006.

COLLIN, E.; PINTO, M. Análise da oferta de construção naval. São Paulo: CEGN - Centro de Estudos em Gestão Naval; Escola Politécnica; USP, 2006. 
COOPER, M. C.; GARDNER, J. T. Building good business relationships: more than just partnering or strategic alliances. International Journal of Physical Distribution \& Logistics Management, v. 23, n. 6, 1993.

CORREA, A. A organização da produção da indústria da construção naval brasileira. Dissertação (Mestrado). Programa de Engenharia de Produção, Universidade Federal do Rio de Janeiro (COPPE), 1993.

COUTINHO, L.; SABBATINI, R.; RUAS, J. Documento: forças atuantes na indústria. NEIT-IE-Unicamp. PR-011 PROTRAN - Programa Tecnológico da Transpetro. Centro de Estudos em Gestão Naval. Escola Politécnica da Universidade de São Paulo, Departamento de Engenharia Naval e Oceânica. São Paulo, 2006.

CRAGGS, J., BLOOR, D., TANNER, B e BULLEN, H. Naval CGT coefficients and shipyard learning, 2004.

DAVENPORT, T. Putting the enterprise into the enterprise system. Havard Business Review, v. 76, n. 4, p. 121-131, 1998.

DE NIGRI, J.; KUBOTA, L.; TURCHI, L. Inovação e a indústria naval no Brasil. Agência Brasileira de desenvolvimento industrial, 2009. Disponível em: < www.abdi.com.br>. Acesso em: 6 fev. 2011.

FERRAZ, J.; KUPFER D.; HAGUENAUER, L. Made in Brazil: desafios competitivos para a indústria. Rio de Janeiro: Campus, 1997.

FOLHA DE SÃO PAULO, edição de 20 abr. 2008. Disponível em: < http://www1.folha.uol.com.br/folha/dinheiro/ult91u393985.shtml >. Acesso em: 24 dez. 2009.

FORTE, S.; MOREIRA, M.; MOURA, H. Competitividade internacional baseada em recursos: estudo do processo de internacionalização das maiores empresas exportadoras do setor calçadista brasileiro. In: ENCONTRO ANUAL DA ASSOCIAÇÃO NACIONAL DOS PROGRAMAS DE PÓS-GRADUAÇÃO EM ADMINISTRAÇÃO. Anais... 2006.

FRAGA, C. Demanda para construção de plataformas offshore. Rio de Janeiro, set. 2002. (Apresentação realizada na Organização Nacional das Indústrias do Petróleo (Onip)).

GONÇALVES, C. Produtividade e instituições no Brasil e no mundo: ensinamentos teóricos e empíricos. In: BARROS, O. ; GIAMBIAGI, F. Brasil globalizado. Rio de Janeiro: Campus, 2008.

GORDON, I.; MCCANN, P., Industrial clusters: complexes, agglomeration and or social networks? Urban Studies, v.37, n.3, p. 513-532, 2000.

GRASSI, A. A indústria naval brasileira no período 1958-94: uma análise histórica de sua crise atual e das perspectivas de mudança a partir do conceito estrutural de 
competitividade. Dissertação (Mestrado) - Departamento de Economia, Universidade Federal Fluminense. Niterói, Rio de Janeiro, 1995.

GREGORY, M.; PLATTS, M. A Manufacturing audit approach to strategy formulation. Proceedings of the 5th Conference of the OMA UK - Manufacturing Strategy. Univ. of Warwick. June 1990.

GROOVER, M. Automation, production systems, and computer-integrated manufacturing. 2.ed. Prentice Hall, 2000.

HAYES, R.;WHEELRIGHT, S.C. Restoring our competitive edge. New York: Wiley, 1984.

HILL, T. Manufacturing strategy. London: MacMillan, 1985.

INTERGRAPH, PRESS REALISE. White paper. an information technology blueprint for the twenty first century amphibious warship, 2004. Disponível em: <http://solutions.intergraph.com>. Acesso em: 10 fev. 2011.

JACOBS, F.; WESTON, F. Enterprise Resource Planning (ERP) - a brief story. Journal of Operations Management, v. 25, n.2, p. 357-363, 2007.

JUNQUEIRA, G. S. Análise das possibilidades de utilização de sistemas supervisórios no planejamento e controle da produção. Dissertação (Mestrado). Escola de Engenharia de São Carlos, Universidade de São Paulo, 2003.

KHANZODE, A.; FISCHER, M.; REED, D.; BALLARD, G. A guide to applying the principles of virtual design and construction (VDC) to the lean project delivery process, CIFE working paper, Stanford University, 2006.

KOTLER, P.; CASLIONE, J. Vencer no caos. Rio de Janeiro: Campus, 2009.

KRAJEWSKI, L.; RITZMAN, L.; MALHOTRA, M. Administração de produção e operações. São Paulo: Pearson, 2009.

LAHNSTEIN, E. Development in the norwegian maritime cluster: first maritime european cluster-organization roundtable.netherlands, april 2004.

LAMB, T. Design for production in basic design, star symposium. Oregon: Portland, 1986.

. Worldwide shipbuilding. In: PALESTRA SOBENA. Rio de Janeiro, 2004. Disponível em:< http://www.sobena.org.br/>. Acesso em: 25 abr. 2005.

LAUDON, K. C.; LAUDON, J. P. Sistemas de informação gerenciais. São Paulo: Pearson, 2004. 
LAURINDO, F.; CARVALHO, M. Changing product development process through information technology: a Brazilian case. Journal of Manufacturing Technology Management, v. 16, n. 3, 2005.

LIKER, J. O Modelo Toyota: 14 princípios de gestão do maior fabricante do mundo. Bookman: Porto Alegre, 2005.

LUTCHEN, M.D. Managing It as a business. Hoboken: John Wiley, 2004.

MELLO, L..; BANDEIRA, R. Lições aprendidas sobre a implementação de sistemas ERP: estudo de casos múltiplos em empresas líderes de mercado, SIMPEP, 2007.

MINTZBERG, $H$. The rise and fall of strategic planning. New York: The Free Press, 1994.

Bookman, 2003. ; AUSTRAND, B.; LAMPEL, J. Safári de estratégia. Porto Alegre:

MOURA, D. A., Análise dos principais segmentos da indústria marítima brasileira: estudo das dimensões e fatores críticos de sucesso inerentes à sua competitividade. Doutorado (Tese). Escola Politécnica, Departamento de Engenharia Naval e Oceânica, Universidade de São Paulo, 2008.

OMER, S.; GÖSLÜ, S. Investment analysis of the cad/cam/cim/cae/cal technologies in the shipbuilding industry. Anais ICPR Americas, 2006.

PEREIRA; N; LAURINDO, F. A Importância da tecnologia da informação na indústria da construção naval: um estudo de caso. Produção, v. 17, n. 2, p. 354-367, 2007.

PETTIGREW, A. Longitudinal field research on change: theory and practice.

Organization Science, v. 1, n. 3, p. 267-292, 1990.

POBA-NZAOU, P.; RAYMOND, L.; FABI, B. Adoption and risk of ERP systems in manufacturing SMEs : a positivist case-study. Business Process Management Journal, v. 14, n.4, p. 530-550, 2008.

REUER, J. \& ARIÑO, A. Strategic alliance contracts: dimensions and determinants of contractual complexity. Strategic Management Journal, v. 28, n.3, p. 313-330, 2007.

SCHOENHERR, T. Outsourcing decisions in global supply chains: an exploratory multi-country survey. International Journal of Production Research, v.48, n. 2, p. 343-78, 2010.

SEDLER, B. Police Maritime Cluster-programs assumptions of modern maritime integrated networks. MARITIME INDUSTRIES FORUM. Anais . GERMANY, January 2005. 
SLACK, N. et al. Administração da produção. São Paulo: Atlas, 2009.

SNIDER, B.; SILVEIRA, G.; BALAKRISHNAN, J. ERP implementation at SMEs: analysis of five Canadian cases. International Journal of Operations \& Production Management, v. 29, n. 1, p. 4-29, 2009.

SPEAR, S.; BOWEN, H.K. Decoding the DNA of the toyota production system. Harvard Business Review, p. 97-106, sept./octo. 1999.

. Learning to lead at toyota. Harvard Business Review, may 2004.

SRIPRASERT, E.; DAWOOD, N. Requirements identification for construction planning and control system, International Council for Research and Innovation in Building and Construction CIB w78 conference 2002. AARHUS SCHOOL OF ARCHITECTURE, 12 - 14 June 2002.

STORCH, R.; HAMONN, C.; BUNCH, H. Ship production: cornell maritime press, Maryland: Centerville, 1995. Improving flow to achieve lean manufacturing in shipbuilding,

Production Planning and control, v. 10, n.2, 1999.

SVENSSON, G. The theoretical foundation of supply chain management - a functionalist theory of marketing. International Journal of Physical Distribution \& Logistics Management, v. 32, n. 9, 2002.

SWINK, M.; MELNYK, S.: COOPER, M.: HARTLEY, J. Managing operations across the supply chain. New York: McGraw-Hill, 2011.

TAKIMOTO, A.K. Proposta de reorganização de um estaleiro público militar: uma utilização do modelo de estrutura divisional. Dissertação (Mestrado) Universidade Federal Fluminense, Niterói, 2005.

THOMPSON, A.; STRICKLAND, A.; GAMBLE, J. Administração estratégica. São Paulo: Mcgraw-hill, 2008.

TORRES, I. (2001). Integração de ferramentas computacionais aplicadas ao projeto e desenvolvimento de arranjo físico de instalações industriais.

Dissertação (Mestrado) -Universidade Federal de São Carlos, São Carlos, 2001.

ÚLTIMO SEGUNDO, edição de 31 ago. 2009. Economia. Disponível em: < http://ultimosegundo.ig.com.br/economia/2009/08/31 > Acesso em: 24 dez. 2009.

VOLLMANN, T.; BERRY, W.; WHYBARK, D. Integrated production and inventory management. Business One Irwin, 1993.

WALL, B. Materials traceability: the à la carte approach that avoids data indigestion. Industrial Management \& Data Systems, v. 95, n.1, 1995. 
WILHELM, K. Collaboration makes construction lean, Target innovation at work, v.23, n.5, p.4-11, 2007.

WOMACK, J.; JONES, D. Lean Thinking: banish waste and create wealth in your corporation. Free Press: New York, 2003.

WU, F.; YENIYURT, S.; DAEKWAN, K.; CAVUSGIL, S. The impact of information technology on supply chain capabilities and firm performance: a resource based view. Industrial Marketing Management, v.35, p. 493-504, 2006.

WU, S.; MELNYK, S.; FLYNN, B. Operational Capabilities: the secret ingredient. Decision Sciences, v. 41, n. 4, 2010.

YIN, R.K. Estudo de Caso: planejamento e métodos. Porto Alegre: Bookman, 2001.

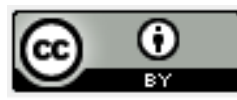

Artigo recebido em 14/04/2010 e aceito para publicação em 10/04/2011. 